msh-mss Mathématiques et sciences humaines

183 | Automne 2008

Hommage à Georges-Th. Guilbaud

\title{
Entretien avec G.-Th. Guilbaud
}

An interview with G. Th. Guilbaud

Denis Bayart

\section{OpenEdition}

Journals

Édition électronique

URL : http://journals.openedition.org/msh/10783

DOI : $10.4000 / \mathrm{msh} .10783$

ISSN : 1950-6821

\section{Éditeur}

Centre d'analyse et de mathématique sociales de l'EHESS

\section{Édition imprimée}

Date de publication : 14 décembre 2008

Pagination : $35-53$

ISSN : 0987-6936

\section{Référence électronique}

Denis Bayart, «Entretien avec G.-Th. Guilbaud », Mathématiques et sciences humaines [En ligne], 183।

Automne 2008, mis en ligne le 15 décembre 2008, consulté le 23 juillet 2020. URL : http:// journals.openedition.org/msh/10783; DOI : https://doi.org/10.4000/msh.10783 


\title{
ENTRETIEN AVEC G.-Th. GUILBAUD ${ }^{1}$
}

\author{
Denis BAYART ${ }^{2}$
}

\section{INTRODUCTION}

Cet entretien avec G.-Th. Guilbaud a été réalisé le 9 novembre 1988 par Denis Bayart dans le cadre d'une recherche menée en collaboration avec Pierre Crépel sur l'histoire des méthodes probabilistes et statistiques dans le domaine des fabrications industrielles, notamment le contrôle de la qualité. Nous avions demandé à G.-Th. Guilbaud de bien vouloir réagir à une série de questions formulées dans le cadre de notre projet de recherche. Bien que n'étant pas lui-même spécialiste de ces questions industrielles, Guilbaud a fréquenté nombre d'hommes d'entreprise et de praticiens qu'il évoque au cours de l'entretien. Il se montre particulièrement intéressé par l'application des raisonnements probabilistes aux problèmes de l'économie et du monde des entreprises, toujours vigilant quant au risque " d'évacuer la probabilité », c'est-à-dire de réduire le raisonnement probabiliste à une procédure déterministe dont le résultat " certain » n'est qu'illusion, voire «hypocrisie». À travers sa conversation au charme inoubliable, il nous offre un panorama très vivant et chaleureux du monde des probabilités et statistiques des années 1930 à 1950.

L'entretien a lieu dans le bureau de Guilbaud, à la Maison des sciences de l'homme à Paris.

Le texte ci-dessous est, pour l'essentiel, une transcription fidèle de la conversation, enregistrée sur magnétophone. Nous avons tenu à respecter les formulations orales de Guilbaud, sachant quelle importance il accordait aux aspects langagiers. Certains passages ont été supprimés, soit très anecdotiques, soit trop centrés sur notre projet de recherche (demande de références précises, de noms, etc...). À plusieurs reprises, Guilbaud a également feuilleté ses propres dossiers et documents, des actes de colloques, etc., pour préciser ses souvenirs - nous ne l'avons pas mentionné ici.

Nous avons également reformulé les interventions de l'interviewer afin de rendre l'ensemble plus compréhensible pour un public élargi, notamment en reprenant l'énoncé des questions que Guilbaud avait sous les yeux et auquel il se référait pour reprendre le fil de l'entretien. Voici la liste de ces questions telles qu'elles étaient formulées à l'époque :

\footnotetext{
${ }^{1}$ Nous remercions Madame Micheline Petruszewycz du soin avec lequel elle a relu ce texte.

2 PREG-Centre de recherche en gestion, École polytechnique et CNRS, Paris, denis.bayart@ polytechnique.edu
} 
- Quand a-t-on posé la question de la maîtrise du hasard dans les processus de production (ingénieurs, marchés...) ? Quand a-t-on conçu que les statistiques pouvaient apporter une réponse ? Comment cette question a-t-elle évolué selon les pays, selon les branches d'activité ? sur des aspects ponctuels et de façon générale ?

- Dans la logique organisationnelle de l'entreprise, comment se développent les statistiques ? Par quels acteurs ? avec quelles formations ? Par quels processus de décision? Quels sont les modes de diffusion au sein d'une entreprise, ou entre branches industrielles?

- Dans quelle mesure le taylorisme a-t-il débouché sur les statistiques? Plus généralement, quelle dialectique s'est développée entre la rationalisation du travail et l'outil statistique ? Quelles conditions a-t-il fallu remplir pour que la mise en place de cet outil soit efficace?

- Quelle a été la place des méthodes statistiques dans les fabrications militaires, selon les services et les armes? Quelles diffusions à travers le monde, quand et avec quels débats?

- Quelle a été l'attitude des clients de toutes sortes (public, armée, privé...) par rapport $\mathrm{au}$ « hasard » et à la qualité des marchandises ? Consulter les textes réglementaires de conditions de recette (armée), de réception (pharmacie...) : conséquences par rapport à une attitude statistique, à une attitude plus mathématique en général ?

- Comparaison entre la démarche du client et celle du fournisseur : dans quelle mesure a-t-on pris conscience de l'existence de cette double démarche, de la contradiction entre ces deux points de vue ? Comment les compromis ont-ils été mis au point, de façon empirique et explicite ou non selon les branches, les pays? Comment le problème a-t-il été énoncé de front ?

- L'utilisation des statistiques dans l'industrie est-elle en retard en France par rapport à celle en Grande-Bretagne, aux États-Unis, en Allemagne ? Si tel est le cas, ce processus a-t-il des racines anciennes ou est-il relativement récent (années 1920...) ?

\section{EN 1946 SE MANIFESTENT DES PRÉOCCUPATIONS AUTOUR DE LA GESTION DU HASARD}

D. BAYART : Notre première question est très générale et vise à cerner des points de repères chronologiques : Quand a-t-on posé la question de la maîtrise du hasard dans les processus de production, quand a-t-on conçu que les statistiques pouvaient apporter une réponse? Qui ont été les acteurs de ces réflexions (ingénieurs, scientifiques, militaires...), en quelles circonstances?

G.-TH. GUILBAUD : Il y a quelque chose qui me frappe. En, disons 1946, je fréquente des milieux qu'on peut appeler économistes, ou économétrie, et statistique. C'est le début de ce qu'on va appeler la recherche opérationnelle, ainsi nommée à cause de ses origines militaires, le mot opérationnel ayant son sens militaire. Une chose qui me frappe à ce moment-là, c'est une agitation intellectuelle assez considérable, dans toutes sortes de milieux : universitaires, ceux que je fréquente le plus. Mais à partir de ce moment-là, je fréquente aussi beaucoup de milieux industriels, qui sont très désireux de... je ne parle pas des milieux militaires - quoique ça vaut la peine aussi. Et je ressens des demandes, des besoins assez considérables.

Je me souviens d'un thème que je développais souvent, dans les milieux d'ingénieurs par exemple, à savoir : l'organisation dite scientifique du travail, Taylor, Fayol et tout le reste - c'est intellectuellement, rationnellement, fort intéressant. Cela pose des problèmes humains, mais c'est une autre affaire. Mais il manque à celle-ci 
quelque chose d'essentiel : l'aléatoire, l'incertain. Et je disais : ce qu'il y a de neuf dans la recherche opérationnelle, c'est uniquement cela, c'est-à-dire la prise en compte du hasard, et les recherches pour donner une certaine rationalité aux conduites humaines devant le hasard. Je ne dis pas de chercher les lois du hasard, ou de dominer le hasard, mais simplement de régler les conduites humaines. Pas mal de laïus que je fais à cette époque s'inspirent de la longue tradition probabiliste depuis Pascal, Laplace, etc. jusqu'à nos jours.

Voilà la première chose qui me vient à l'esprit quand vous dites : « quand a-t-on posé la question... de la maîtrise du hasard dans les processus de production ? ». On l'a posée très tôt, bien sûr. Mais il y a un renouveau, me semble-t-il. Maintenant, je ne suis pas juge puisque je suis partie, comme on dit. Mais je sens très nettement à partir de 1946 un renouveau de ce désir de maîtrise du hasard... avec des difficultés considérables, évidemment.

Il y a des hommes, de grande stature, plus vieux que moi, ou même [seulement] un peu plus vieux que moi, qui sont des leaders aussi dans ce domaine. Deux noms me viennent à l'esprit. Pierre Massé, dont le rôle a été, me semble-t-il, exceptionnel, et on ne le dit pas suffisamment. Pierre Massé était quelqu'un de considérable ; la publication de ce qu'on peut appeler sa thèse, chez Hermann, les réserves de l'avenir (vous devez le lire), sur la régulation des réserves hydro-électriques. Dans Massé, il y a quelque chose d'intéressant : d'abord, la nécessité de faire du calcul des probabilités, et propre. Puis celle de s'inspirer du travail actuariel, parce qu'il se trouve qu'il n'y avait pas beaucoup de contacts entre le milieu des ingénieurs et le milieu des actuaires. Si j'écrivais mes mémoires, ce qu'à Dieu ne plaise, j'essayerais de raconter le bouillonnement d'idées entre planification (pas de doute, Monnet et le reste, la planification commence à s'installer en France), la comptabilité nationale... et, ce qui est très important, il y avait une sorte de clivage, entre les gens pour qui la maîtrise de l'aléatoire - je dirais plutôt, comme Massé, la gestion de l'aléatoire, parce que la maîtrise de l'aléatoire, ça donne des idées superstitieuses et vaines - c'est : « on n'y peut rien, nous sommes embarqués, peuton donner plus de rationalité aux conduites grâce aux schémas probabilistes ? », toute la question est là ; alors la réponse était à cette époque-là très fortement... Un homme aussi très important dans ce domaine, c'est Gabriel Dessus - vous n'avez jamais entendu parler de cet homme... la géographie volontaire. Il a été un homme important à EDF, il n'a pas écrit beaucoup, c'était surtout un homme de paroles et un homme d'action.

Dessus, Massé, tous ces gens qui complètent l'idée de planification par la mise en garde permanente : " faites attention, beaucoup de choses - on ne dit pas : tout - en ce bas monde sont incertaines, il faut faire avec ». Le problème, c'était : " peut-on utiliser les schémas probabilistes? »Et aussi bien Massé que Dessus insistaient beaucoup c'étaient aussi les débuts de Boiteux, et de Debreu - la réponse est : " pas toujours ». Car toute incertitude n'est pas probabilisable. C'était le maître-mot.

Voilà comment les choses, à mon avis, ont commencé. Les actuaires et les ingénieurs, le calcul des probabilités et son usage industriel, les défauts de l'organisation scientifique du travail, et aussi d'ailleurs de la planification économique, s'ils ne tiennent pas compte des aléas...

C'était la réponse à votre première question. Ce n'est pas une réponse, d'ailleurs... «Quand a-t-on conçu que les statistiques pouvaient apporter une réponse...? ». Je pense : tout le temps; je ne vois pas que ce soit une innovation. 
COMMENT G.-TH. GUILBAUD EN EST VENU À ÉTUDIER LES PROBABILITÉS DANS LES

ANNÉES 1930 ?

D. BAYART : Si on se place dix ans ou vingt ans auparavant, était-ce la même chose ? Dans les débats de X-Crise par exemple, était-il question du hasard ?

G.-TH. GUILBAUD : Ah ! dans les années trente... Je ne suis pas bien vieux : X-Crise, c'est des gens de la génération avant moi. Je lis leurs prospectus en 1932-1933, ça m'intéresse, mais enfin je suis quand même débutant. À ce moment-là, d'ailleurs, ma carrière universitaire c'est les mathématiques; pas question de faire autre chose. J'ai même hésité - ce sont des accidents de ma biographie personnelle qui ont fait que je n'ai pas fait de mécanique, ce qui était mon but. Quand je me suis lancé dans la statistique, c'était de la mathématique.

Qu'est-ce que les gens de mon entourage pensaient du hasard et du calcul des probabilités ? Que ça existait... Il y avait universitairement un certain mépris. Il est bien clair que ce n'est pas ce que l'on faisait de mieux. J'ai à peu près l'âge de Bourbaki, voyez. À ce moment-là, le calcul des probabilités, la statistique... Et ce n'est pas parce qu'elles sont appliquées, car la mécanique c'est très bien [considéré]...

D. BAYART : Mais vous-même, faisiez-vous de la statistique mathématique, ou d'autres mathématiques?

G.-TH. GUILBAUD : Je n'avais pas l'intention de faire de la statistique, et il s'est trouvé que des nécessités impérieuses de gagner ma vie tout de suite, la mort de mon père enfin peu importe... - ça c'est de la biographie, et je n'aime pas la biographie, comme vous le savez... J'ai pris, parce que c'était possible... Il fallait que je me dépêche d'avoir tous les diplômes nécessaires pour sortir ; j'étais en retard, et il y avait ce diplôme de statistique mathématique chez Darmois ; " vas-y, c'est pas difficile, tu verras, tu auras ça en un mois ou deux ». C'est ce que j'ai fait, j'ai passé l'examen. Et puis, je me suis dit: « Bon Dieu, mais c'est intéressant, ce truc-là ! ». Alors je l'ai gardé dans ma petite tête, dans un coin. Je l'ai gardé... entre 35 et 39 , où j'étais professeur de taupe - ce qui ne prédispose pas à faire du calcul des probabilités, comme vous le savez... Mais je m'intéressais à la statistique, et surtout à l'économie mathématique, et je voyais des liens entre les deux.

Quelle était l'opinion publique générale ? Peut-être pas très... en effet... faudrait voir... les écrits d'un homme qui a été assez important dans ce domaine, c'est René Roy. Et aussi peut-être Divisia.

D. BAYART : Puisque vous évoquez Divisia, cela me rappelle qu'il militait en faveur des statistiques. Il a d'ailleurs adressé au Laboratoire de probabilité de Fréchet, pendant la mobilisation scientifique de 1939-1940, une question sur "l'homogénéisation des bouquets ou lots d'utilisation ", qui semble avoir un rapport avec le contrôle de la qualité, et qui a été confiée à Halphen. Divisia était alors au Ministère des travaux publics. Savez-vous quelque chose à ce sujet ? Il y a eu également quelques autres questions, venant soit de l'Armée, soit d'entreprises travaillant pour l'Armée.

G.-TH. GUILBAUD : Oui, sans doute, mais Roy est d'une tout autre stature. Le labo de Fréchet, c'était un truc un peu « comme ça », ce n'était pas vraiment institutionnel, mais c'était anecdotiquement très important. Le labo de Fréchet était aussi, il ne faut pas l'oublier, un refuge pour étrangers.

C'est dans ce labo de Fréchet - j'y suis allé une fois ou deux, j'étais mobilisé moi aussi à l'État-major, et je venais quelquefois à Paris pour des questions d'ordre 
scientifique. Je me souviens - c'est une des choses qui m'ont beaucoup frappé - qu'ils étaient en train de redécouvrir le problème de Monge. C'est très important parce qu'à la même époque, et aux États-Unis et en Union Soviétique, on phosphore comme des dingues sur la question du problème du transport. Mais c'est le problème de Monge ! Parce que Monge l'a posé comme un militaire. C'est celui des fortifications, des déblais et des remblais, c'est la première idée d'organisation scientifique du travail. Nous somme en 1775 ou 80, à l'École du génie de Mézières, le jeune Monge pose le premier problème vraiment important, et difficile puisque c'est le problème du transport: comment minimiser le coût des transports, connaissant tous les points de départ et tous les points d'arrivée. Évidemment, quand il n'y a qu'un seul point de départ et d'arrivée, il n'y a pas de problème ; mais quand il y en a un grand nombre, que faire ? Monge pose le problème, et c'est une aventure intellectuelle extraordinaire. D'abord parce qu'elle est de très longue durée - pendant plus de 150 ans, il y a ce problème bien posé, et la plupart des usagers potentiels ne se rendent pas compte de ce qu'il y a derrière. Ils transfèrent il faut voir les livres concernant les travaux publics sur tout le XIX ${ }^{\mathrm{e}}$ siècle. Tout le monde cite ce qu'ils appellent la « règle de Monge » : il ne faut pas que les brouettes se croisent. Ça, bon. Mais en même temps, personne ne fait le lien avec ce que Monge luimême a écrit sur la question, qu'il a appelé problème des déblais et des remblais. Il n'a été utilisé qu'en optique géométrique - mais c'est un truc magnifique des mathématiques. C'est les caustiques de Malus et Dupin. Quand j'étais petit, j'ai appris ça, c'est très marrant : la congruence des normales, il y a de quoi jouir, là-dessus. Mais personne ne me disait quelle était l'origine, c'est-à-dire déblais et remblais ; c'est-à-dire un problème « un maximum se traite par le potentiel », c'est aussi bête que ça. Pire, en 1930, ou 29, Paul Appell, probablement à la retraite à ce moment-là, publie dans le Mémorial des sciences mathématiques un fascicule - ces fascicules rouges qui ont fait la gloire de la mathématique française - sur le problème des déblais et remblais. Lisez la préface, il dit : «oh, déblais et remblais... : on croit ça parce que Monge était en effet capitaine du génie, ça n'a aucun intérêt pratique, mais du point de vue mathématique, c'est une petite merveille ». Il dit, en 1930 : «ça n'a aucun intérêt pratique ». C'est pourquoi, en 38-39, sous la pression des militaires peut-être, on a retrouvé le problème, en ignorant complètement que Monge l'avait traité, et c'est devenu la programmation linéaire, pour dire les choses comme elles sont.

D. BAYART : Vous rappelez-vous qui travaillait sur cette question ?

G.-TH. GUILBAUD : Un gars chez Fréchet, qui était un étranger; je ne me rappelle plus sa nationalité, c'est à vous de la trouver. Sur le moment, d'ailleurs, cela ne m'a pas frappé, car je n'étais pas au courant - personne ne m'avait expliqué ces choses-là. Ce n'est qu'après, quand j'ai découvert à la Libération les travaux de Kantorovitch et de Dantzig, que j'ai vu que c'était de ça qu'il s'agissait. Comme c'est cela que j'enseignais à la même époque, je suis revenu en arrière. Mais ce n'était pas probabiliste.

\section{LES RELATIONS DE LA STATISTIQUE AVEC L'ÉCONOMIE}

D. BAYART : Vous évoquiez tout à l'heure les relations de la statistique avec l'économie mathématique. Étiez-vous le seul à les voir, à cette époque?

G.-TH. GUILBAUD : Oh non! Quand j'ai commencé d'enseigner la mathématique pour les économistes, dans ce qu'on appelait à l'époque les facultés de droit et appelées un peu plus tard «facultés de droit et de sciences économiques ». Je ne suis pour rien dans cette modification, mais elle était dans le sens de... Mes collègues, c'est-à-dire les profs d'économie des facultés de droit, d'ancien style si vous voulez, n'étaient pas tous 
méprisants. Il y avait une petite tradition, des gens comme Aftalion, Jean Marchal, Henri Guitton, ils étaient favorables ; bien sûr, ils bricolaient, une statistique pas très savante. Mais l'influence des statisticiens-économistes anglo-saxons était considérable ; les grands travaux de Mitchell sur les cycles économiques étaient connus, étudiés. Ils n'avaient pas beaucoup fait de mathématique, ils n'étaient pas très au courant, le langage mathématique était pour eux difficulté, mais enfin... On a une bonne idée de l'état de choses en lisant le gros traité de Lucien March. Ce n'était pas totalement méprisable, c'était quelque chose d'important. Chez les économistes des travaux de ce genre étaient considérés, pour parler comme les historiens, comme des sciences auxiliaires. Il était absolument indispensable de manier du chiffre, et pour manier du chiffre, il fallait quand même prendre des gants et des instruments, parce que la statistique, c'était plus savant qu'on ne le disait. Voilà à peu près l'idéologie de l'époque.

D. BAYART : En parcourant March, j'ai eu l'impression que c'était plutôt des statistiques descriptives, qu'il y a assez peu d'aléatoire.

G.-TH. GUILBAUD : C'est exact. Il y a une partie de statistique descriptive, elle est indéfectible, même encore de nos jours. Il y a deux endroits où l'aléatoire affleure, c'est l'idée de corrélation et les séries chronologiques. C'est pour cette raison que j'avais écrit - plus tard, entre 50 et 60 - un petit bouquin que j'ai appelé Statistique des chroniques. Vous pouvez le prendre comme un document historique, témoignage de l'enseignement élémentaire de la statistique dans les milieux économistes. Parce qu'il se trouve que, par suite d'accidents historiques, j'ai enseigné, non pas dans le cadre des facultés des sciences, mais dans celui des facultés de droit.

C'est dans ces années 50-60 que j'ai produit quelque chose. Avant, je n'avais pas autorité pour parler. Mais il y avait une certaine ouverture de la part d'un petit nombre d'économistes. Évidemment, un refus d'un assez grand nombre.

Il faudrait aussi parler des milieux d'administration, des affaires, de la banque, de la finance...

\section{L'ENSEIGNEMENT DES STATISTIQUES DANS LES ANNÉES 1930}

D. BAYART : Où enseignait-on la statistique à l'époque ? Il y avait l'ISUP, mais aussi le CPA, le "Centre de préparation aux affaires », et on peut penser que des praticiens y enseignaient. Par exemple Ernest Lhoste, officier d'artillerie, économiste et directeur de l'AFNOR de 1938 à $1947^{3}$.

G.-TH. GUILBAUD : L'ISUP, c'est compliqué. C'est une aile, une machine latérale de la Faculté des sciences. Mais il y avait le cours de Darmois à la Faculté des sciences de Paris. C'était un certificat de probabilité et de statistique mathématique, dont le témoignage de l'enseignement est son célèbre livre - j'espère que vous le connaissez publié chez Doin en 1928, dans l'encyclopédie de Thoulouze. C'est par là que je suis entré en contact avec la statistique. C'était enseigné à la Faculté des sciences de l'Université de Paris, indépendamment de toute préparation aux affaires et de l'ISUP.

\footnotetext{
${ }^{3}$ Cf. l'article : Nacira Hadjadji Seddik-Ameur, «Les tests de normalité de Lhoste », Mathématiques et Sciences humaines 162, été 2003, [mis en ligne le 10 février 2006 : http://msh.revues.org/document2886.html].

Cf. également l'article : Lyle Broemeling, Ana Broemeling, «Studies in the History of Probability and Statistics XLVIII, the Bayesian Contributions of Ernest Lhoste », Biometrika 90(3), septembre 2003, 728731.
} 
D. BAYART: Je citais ceux-ci en rapport avec les applications de la statistique à l'industrie. Y avait-il beaucoup d'industriels à la Faculté des sciences ?

G.-TH. GUILBAUD : Tout passait par Darmois. Il ne faut pas oublier que Darmois était un industriel, il avait une petite forge, de quelques ouvriers, dans les Vosges. Il avait beaucoup de relations à Paris avec les milieux d'affaires. Il plaisantait souvent en effet sur le fait que ses collègues fréquentaient peu les milieux d'affaires et qu'ils avaient tort.

D. BAYART : Il s'intéressait donc aux applications...

G.-TH. GUILBAUD : Le contrôle de fabrication, votre sujet, je n'ai pas grand chose à en dire car je n'ai pas vraiment connu. C'est essentiellement les économistes, le Commissariat au Plan, l'EDF et la SNCF : la gestion... en gros. Le contrôle de fabrication, j'en ai entendu parler, bien sûr. Les premières publications qui ont eu de l'importance, l'une signée de Darmois, les autres de Mothes, je crois, les grands cahiers verts de l'Imprimerie nationale, ils doivent être à [la bibliothèque de] l'INSEE. Il y a trois fascicules, de 46-47. C'est le début de la propagande pour les cartes de contrôle.

D. BAYART : Cependant, les cartes de contrôle, c'est plus ancien...

G.-TH. GUILBAUD : Oui, mais pour propagander, il faut que ça existe. Et puis il faut bien se rendre compte de la coupure de 40-45, qui est quelque chose d'énorme.

\section{PENDANT LA GUERRE, LA STATISTIQUE CLANDESTINE}

D. BAYART : Mais ne s'est-il rien passé pendant la guerre sur le plan des statistiques ?

G.-TH. GUILBAUD : Si, il s'est passé des tas de choses dont je ne connais pas les détails. L'histoire n'a jamais été faite... Sauf peut-être à propos de la biographie de Carmille, on a dû raconter... Il y a eu un travail statistique clandestin considérable. Ça peut paraître bizarre, la statistique clandestine... mais c'est là que j'ai découvert - ou cru découvrir une idée qui a par la suite été plus ou moins développée, contestée ou exploitée... En tout cas je le disais comme ça à l'époque: la statistique, c'est essentiellement la conscience qu'une société se donne d'elle-même. Vous voyez que c'était tendancieux. Je veux dire par là qu'on ne peut pas projeter sur une société déterminée la statistique comme... Voyez les réflexes qui ont été acquis pendant l'Occupation : quand on faisait une enquête, eh bien ! on se défilait. Par contre, et j'avais eu contact à ce moment-là avec des militants ouvriers de la région de Lyon-St Étienne, n'importe quel milieu social a intérêt à se connaître lui-même. Lien d'ailleurs à l'idéologie, pourraient dire les historiens futurs, avec la JOC dont c'était l'un des thèmes fondamentaux. Ça avait été une redécouverte que se connaître. Je ne sais plus quel était leur slogan, ça pouvait prendre une allure un peu scientifique, et même qu'il valait mieux... Je me souviens en particulier d'une enquête qui avait été faite en Région parisienne, mais j'ai perdu la trace... j'ai perdu les gens de vue, maintenant il me revient des souvenirs... Il y a eu aussi l'influence d'Économie et humanisme, influence marginale, de la même inspiration : estce que la statistique c'est pour les polytechniciens, ou est-ce pour le peuple ? Réponse : oui, le peuple a droit à une statistique qu'il fera lui-même. Un thème, naïf peut-être, qui a pris conscience de lui-même à la Libération, mais qui a joué un certain rôle pendant l'Occupation. L'idée de réseau est statisticienne et sociologique à la fois.

Il me revient progressivement des souvenirs, mais il y a longtemps que je n'en ai plus parlé, que je n'y ai plus pensé : sur ce qu'on peut appeler l'idéologie qui emballe toutes ces choses. Idéologie qui était née d'une expérience vécue particulièrement dramatique, qui ne laissait pas les gens indifférents. En particulier, je me souviens 
d'avoir fait des laïus sur les divers sens du mot « réseau », qui a un substrat. J'avais été très frappé, d'ailleurs, de voir que les problèmes de clandestinité méritent d'être un peu éclairés par une réflexion, y compris de type mathématique. Je me suis aperçu après, en 45-46, qu'il y avait eu des travaux, stipendiés par des militaires, dans ce domaine ; à savoir : les réseaux de communication, et quand ils sont coupés, qu'est-ce qui se passe ? $\mathrm{Ca}$ a donné lieu à une littérature très abondante, mathématicienne en diable, et on a cessé d'appeler ça des réseaux, on a appelé ça des graphes - ça n'a pas été un progrès à mon avis, mais enfin... Tout ça a bouillonné, et il y a eu une statistique clandestine.

D. BAYART: Il y a des informations dans le livre de Michel Volle ${ }^{4}$, et dans les témoignages recueillis par François Fourquet ${ }^{5}$.

\section{AUTOUR DE LA GUERRE : SOUVENIRS SUR WALD, BOUZITAT, DARMOIS}

D. BAYART : À propos de documents produits pendant la guerre, connaissez-vous les travaux du Statistical Research Group de Columbia?

G.-TH. GUILBAUD : Non, je ne les ai jamais eus en mains. Mais cela m'amène à parler de Wald. La découverte, à la Libération, disons en 45-50, d'Abraham Wald a été un événement considérable. Et je me souviens encore qu'on avait invité Wald à faire des laïus à Paris - c'était Darmois qui l'avait invité. Oui, je l'ai vu, Wald, c'était un homme très remarquable : un peu tout rond, petit, Viennois en diable, parlant toutes sortes de langues, avec des accents indescriptibles à chaque fois, mais parlant bien... Je me souviens vaguement, dans le brouillard (je n'ai pas une très bonne mémoire historique), de conversations avec Darmois à propos du papier de Bouzitat sur les travaux de Wald, et sur l'importance, selon Darmois, qu'il y avait - et voyez, ce n'est pas idiot, puisqu'un homme tel que Wald le fait - à associer les tactiques bayesiennes et les procédures séquentielles. C'étaient là les deux clés. Et c'est vrai que Bouzitat l'avait redécouvert tout seul, à l'ONERA ${ }^{6}$, je ne sais comment. Alors, à propos de Bouzitat, prenez ses coordonnées, et il faut que vous alliez le voir. Ce document (l'Annuaire des anciens élèves de Normale Sup), pour un historien, est intéressant aussi... y compris l'analogue pour l'École polytechnique, mais ça vous devez l'avoir... Parce que Bouzitat ne m'a jamais raconté à la suite de quelle occasion il avait écrit ce mémoire. Il pourra raconter, je pense... En tout cas, ça nous avait paru, à l'époque... je me souviens, Darmois en faisait grand cas. Il espérait que ça serait... que bien d'autres gens travailleraient dans ce sens-là. Il semble bien que Bouzitat ait découvert ça tout seul. Mais il l'a écrit tout de suite à la Libération - l'ONERA a été fondé à la Libération. Comment il est entré à l'ONERA, je ne le sais pas.

D. BAYART : J'aimerais savoir ce qui s'est passé pendant la guerre : Fréchet, Darmois...

G.-TH. GUILBAUD : Darmois était à Londres, moi je n'y suis jamais allé, et je n'ai pas eu de contacts avec lui. Il y était comme scientifique-militaire, moitié-moitié. S'il était à Londres, ce n'était pas un choix comme ça; il avait été envoyé à Londres - il était mobilisé - pour une mission scientifique de contact avec les Britanniques, et il est resté là-bas. Un homme qui doit savoir ça, qui connaissait bien Darmois, c'est Maurice Giraud, qui a été professeur à Paris I. Il a écrit des tas de petites choses de statistiques et de probabilités, et il a terminé à Paris I. Comme Bouzitat, il faisait partie...

\footnotetext{
${ }^{4}$ Volle M., « Histoire de la statistique industrielle », Economica, Paris 1982.

${ }^{5}$ Fourquet F., «Les comptes de la puissance. Histoire de la comptabilité nationale et du Plan », Coll. Encres, éditions recherches, Paris, 1980. Des travaux sur Carmille et la statistique clandestine ont été publiés depuis cet entretien, cf. les références de l'article sur René Carmille dans Wikipedia (FR).

${ }^{6}$ Bouzitat J., Note sur un problème de sondage, ONERA, publication n ${ }^{\circ} 1,1947$.
} 
Dans les années soixante, je dois avouer que j'ai beaucoup intrigué pour faire nommer un certain nombre de gens que je connaissais, en général un peu plus jeunes que moi, qui savaient des mathématiques, dans l'enseignement économique pour donner un petit peu d'air à cette affaire-là. Alors, il y avait Giraud, Bouzitat, Kreweras... En cherchant des gens qui avaient des connaissances de la probabilité... un peu sérieuses. Parce que je me suis engueulé un certain nombre de fois avec certains de mes collègues mathématiciens... « professionnels » comme disait mon ami Dieudonné, c'est-à-dire « de carrière » (rire), concernant les probabilités. Aucun n'a de grande estime pour les probabilités, aucun de ces grands messieurs. Surtout après 68, lors de la refonte des méthodes de recrutement universitaire. J'ai siégé dans un certain nombre de commissions de ce genre, et... je regardais les dossiers, bien sûr et je disais : « ces deux candidats, bon, il s'agit d'un mathématicien, mais d'un mathématicien qui sera en contact avec des économistes, des psychologues, etc., ça serait tout de même bien qu'il ait des lumières sur les statistiques et les probabilités... - « Oh, bien sûr, mais ça s'apprend pendant les vacances !... ». C'était leur réaction spontanée, des gens qui considéraient que ça n'existait pas. En effet, d'un point de vue mathématique, en un certain sens, c'est petit, mais tout le problème est de savoir quel peut être le contact de cette mathématique-là avec une réalité qui est économique, ou démographique... Et ça, ça ne s'improvise pas!

ON NE «MAÎTRISE » PAS LE HASARD, MAIS ON DOIT EN TENIR COMPTE DANS LA GESTION

G.-TH. GUILBAUD : Revenons à vos questions : Comment cette question de la maîtrise du hasard a-t-elle évolué selon les pays, selon les branches d'activité ? sur des aspects ponctuels et de façon générale ?

Moi, je ne dirais pas «maîtrise du hasard » mais gestion du hasard. Et si j'avais à écrire quelque chose dans ce schéma, je commencerais par lire Massé, le grand bouquin de Massé, sur la gestion des réserves de l'avenir...

D. BAYART : Oui, nous en avons déjà parlé. Et Divisia, qui a publié «Statistique et technique » ? Il donne le tableau de gens qui travaillaient chacun dans leur coin, avec des méthodes bricolées, peu rigoureuses, rien de comparable à ce qui pouvait se passer aux États-Unis ou en Angleterre. C'était assez peu institutionalisé. Aux États-Unis, il y avait l'équipe de la Bell, qui a commencé dès les années 1920 à publier des travaux en interne, et même dans le Journal du système Bell. Il semble que les Allemands aient fait des choses dans les années 1920, puisque March, en 1930, parle du contrôle de fabrication en 4 pages, et il prend ses exemples dans un livre allemand de $1927^{7}$. Ensuite, ce sont les Anglais qui s'y attaquent, vers 1932-35, avec de nombreuses publications dans le Journal of the Royal Statistical Society.

G.-TH. GUILBAUD : Il y a eu un phénomène qui a certainement joué un grand rôle dans l'aventure de l'application des statistiques à l'industrie : c'est la bière Guinness. Gosset, Student pour ne pas compromettre la maison. Le $t$ de Student est né d'une recherche véritable, authentique, de Gosset, sur... je ne sais pas, la levure de quelque chose, ou sur les pommes de terre irlandaises, enfin sur quelque chose de réel. C'était en 1904 ou 1905. Voir sa biographie dans le recueil d'articles historiques de Biometrika.

\footnotetext{
7 R. Becker, H. Plaut, I. Runge, Anwendungen der mathematischen Statistik auf Probleme der Massenfabrikation, Berlin, Julius Springer, 1927. (2 éd. 1930).
} 


\section{LE TAYLORISME ET LE REJET DE LA PROBABILITÉ}

G.-TH. GUILBAUD : Continuons avec vos questions : « dans quelle mesure le taylorisme a-t-il débouché sur la statistique ? » Il n'a pas débouché, justement. À mon avis et en tant que «dans le bain », il y avait dans le taylorisme un refus de penser l'aléa qui a été caractéristique. Ce n'était pas [une propriété spécifique au] taylorisme, mais il y a toujours un refus régnant dans la mentalité occidentale, depuis deux ou trois siècles... $C^{\prime}$ est très dur : comme on disait vendredi dernier [au séminaire]... « quelque chose sort de l'intervalle de confiance... il s'est gouré ! ». «Il ne s'est pas gouré, mon bon monsieur, il a perdu son pari : c'est tout à fait autre chose ». " Mais si la probabilité est d'un milliardième, ne me dites pas... ». « Mais si, mon bon monsieur, je le dirai, car si vous ne le dites pas, vous évacuez la probabilité ». Alors ça, on n'y pourra rien, et il y en a peut-être encore pour des siècles. Il y a un refus de la probabilité et de la probabilisation dans la mentalité ambiante. Ça se dissimule de diverses façons. Il y en a qui sous la houlette - hélas ! de Borel (il faudrait éclairer d'ailleurs, cette affaire, c'est dans la biographie personnelle de Borel, probablement) - disent : si les probabilités sont vraiment très petites, en 10 puissance -48 , alors on peut les négliger. Faites attention : comment, en mathématiques, peut-on quelque part dire qu'un nombre est totalement négligeable? Si c'est un produit fini, oui, mais si vous le soumettez au calcul, non. Toute l'analyse en mathématiques s'est construite à partir du $\mathrm{XVI}^{\mathrm{e}}$ siècle sur ce refus du négligeable en tant que tel. D'où la querelle des indivisibles, etc. Il a fallu 100 ans pour que le langage de Leibnitz, passant par Lagrange et par Euler, arrive à un statut qui soit raisonnable. C'est difficile, de parler de l'à-peu-près. Mais il faut en parler rigoureusement. Voilà tout le noeud de la question, pour moi, maintenant.

D. BAYART : Borel a-t-il eu une influence et dans quel sens ?

G.-TH. GUILBAUD : Une influence dans le genre de : flatter l'opinion dominante. Pas toujours d'ailleurs, en particulier pas dans ses travaux de jeunesse qui sont quand même très remarquables. Le mémoire sicilien, c'est une merveille. C'est vrai que l'invention des probabilités dénombrables, et la rigueur dans ce domaine, c'est prodigieux. Mais par la suite, quand il commence à devenir un académicien, un homme qui écrit dans la Revue des deux mondes, etc. et qui écrit pour le grand public, il tombe dans une philosophie du hasard qui est pernicieuse à mon avis. Pas toujours, parce que c'était quand même un brave homme, c'était un homme honnête... malgré que c'était aussi un homme de pouvoir. Tout n'est pas mauvais, mais il a la plume trop facile... à partir d'un certain âge, il écrit à coups de ciseaux. Ce n'est pas très sérieux. problème.

Alors, le taylorisme n'a pas débouché sur les statistiques, et c'est là qu'est le

D. BAYART : Le taylorisme, non, mais la recherche opérationnelle qui a été développée pendant la Deuxième guerre mondiale fait bien une place au hasard. Quels rapports peut-on voir entre les deux, en les considérant comme des formes de rationalisation du travail?

\section{LES PROBLÈMES DE VOCABULAIRE POUR PARLER DU HASARD « SÉRIEUSEMENT »}

G.-TH. GUILBAUD : «Une place au hasard », vous parlez mal, là. Il ne faut pas dire ça. Si vous dites ça, vous êtes foutu, on ne vous écoutera pas. Il faut que le mot de hasard ne soit pas même prononcé. Il faut trouver un vocabulaire... Je me souviens des efforts avec Dessus et avec Massé, aux alentours des années 50. Le problème, surtout avec Dessus qui était beaucoup plus agitateur, quand on faisait des conférences à l'étranger... 
C'est l'EDF qui était motrice, la SNCF un peu moins, beaucoup moins même. Pourquoi l'EDF ? À cause de la pluie et du beau temps, du froid et du sec, etc. La production hydro-électrique est forcément aléatoire. Il faut se colleter avec ça. Mais comment fautil en parler? Je me souviens d'avoir été avec Dessus aux Galeries Lafayette, vers 194850, pour essayer de les convaincre que, dans un truc comme ça, il peut y avoir des catastrophes, et qu'il est bon de les prévoir. «Tu vas voir, me disait-il, l'impossibilité qu'il y a à faire accepter [l'idée de hasard]... ». «Il faut éviter ça à tout prix », disaientils. On relevait cette phrase : «à tout prix». «Attention, nous sommes des économistes : quel prix ? ». Même chose d'ailleurs dans les discussions sur la sécurité des barrages, en particulier les premiers barrages-poids dont Serre-Ponçon. Je me souviens des discussions à ce propos : "S'il pète, ça fera du bruit jusqu'en Avignon ; est-ce qu'on peut probabiliser ?... ». C'était tout un mythe, qui rendait sensible aux gens qui savaient du calcul des probabilités la difficulté qu'il y a de parler, spécialement aux hommes responsables, aux décideurs comme on dit, de parler un langage adapté, qui ne soit pas le langage farfelu du hasard, de la chance, etc. Le mot « risque » était un peu meilleur. La redécouverte de la théorie du risque - là encore, c'est Massé - vers 1950 ; il faut savoir que la théorie du risque existait depuis plus de cent ans, mais qu'elle avait pris des formes qui n'étaient plus connues que des actuaires.

Je suis allé deux ou trois fois à la société des actuaires, c'est vraiment un monde très cléricalisé, très fermé. Mais eux savaient du calcul des probabilités. Ah ! Un homme important, peut-être : Dubourdieu. Un autre, mais plus vieux, moins novateur : Galbrun. Dubourdieu était plus théoricien, justement, se rendant compte de la difficulté qu'il y a à parler proprement des probabilités quand on est en contact avec une réalité. Comprenez, c'est ça toute la question : le seul domaine où la probabilisation soit acceptée, c'est les jeux de hasard, et si possible de hasard pur. Malgré les efforts de Borel pour dire : attention, depuis Bernoulli, il y a aussi les jeux d'adresse, il y a du hasard dedans. Mais les jeux, et l'idéologie du jeu ont joué un rôle considérable, et à mon avis pas toujours excellent, dans la diffusion des idées probabilistes. C'est comme la « théorie des jeux »... j'ai bien été obligé de parler comme tout le monde, mais cette étiquette est épouvantable. Il aurait fallu essayer de la remplacer par autre chose, mais on n'a pas réussi.

On n'a pas réussi parce que... Il faut bien se rendre compte que c'est un problème philosophique qu'il ne faut pas aborder philosophiquement, mais il faut reconnaître d'abord que c'est un problème philosophique considérable, à savoir : le contact d'une théorie mathématique avec une réalité vivante et complexe, comme sont les phénomènes économiques et sociaux. C'est là, le problème de la statistique. Et ce n'est pas simple... c'est extrêmement dangereux. Donc, le problème du vocabulaire... Darmois était très attentif sur ces questions. Il y a un homme moins connu, un peu plus effacé mais quand même très actif, Morice, qui a joué un grand rôle dans cette affaire, et lui aussi se préoccupait beaucoup du vocabulaire. C'est lui qui a dirigé l'école d'application [de l'INSEE] pendant fort longtemps, peut-être même dès sa fondation.

D. BAYART : Peu après la Libération, Fréchet a animé dans le cadre de l'AFNOR un groupe de travail sur la terminologie des statistiques. Ce groupe se souciait aussi du vocabulaire...

G.-TH. GUILBAUD : Oui, mais là c'est le vocabulaire au sens de l'AFNOR. Moi je dis : le vocabulaire au sens de la publicité. Comment faut-il parler pour convaincre ?... Je me rappelle cette réunion aux Galeries Lafayette, malheureusement je n'ai pas gardé de notes, mais on en ferait un beau petit film : le directeur des Galeries, son staff autour de lui, et puis Dessus, moi, et quelqu'un d'autre, essayant de leur dire: le calcul 
économique, ça existe, et surtout en cas de risque considérable. C'est le problème des risques majeurs, quoi, problème qui est encore vivant maintenant, à propos des centrales nucléaires, etc. Il me revient un autre nom : Morlat. Il sait des choses, il a vécu pas mal de choses. L'EDF a joué un rôle capital au point de vue intellectuel dans ce domaine, il n'y a pas de doute, et principalement avec la dynastie Dessus, Massé, Boiteux, plus tous leurs collaborateurs.

\section{LES STATISTIQUES DANS L'ARTILLERIE : DES RÉFLEXIONS THÉORIQUES PROFONDES}

\section{ALIMENTÉES PAR DES SUJETS PRATIQUES}

G.-TH. GUILBAUD : Reprenons vos questions. "Quel a été l'impact des méthodes statistiques dans les fabrications militaires, selon les armes?» Ah! L'artillerie indéniablement, il y a une longue tradition. Il y a de longues traditions, comme ça, longues dans la durée : celle des artilleurs, celle des actuaires. Et je constaterais, si j'étais historien, qu'elles diffusent peu à l'extérieur de leur petit milieu. Et alors, peutêtre plus [encore] l'artillerie navale.

D. BAYART : Alors ce ne serait pas un hasard si les premiers articles français ont été écrits par Maurice Dumas, ingénieur de l'artillerie navale?

G.-TH. GUILBAUD : Oui. Mais je n'ai pas beaucoup d'informations sur ces questions, quoique j'aie eu deux oncles qui ont terminé leur carrière comme ingénieurs généraux là-dedans. J'ai souvent eu en main des documents, c'était quand même du travail sérieux. Les débats, même. Tenez, pendant la guerre, en 1940, chargé de je ne sais plus quoi - je tirais contre les avions - et on était en train d'inventer ce qu'on appellera plus tard le radar (là aussi il y a des problèmes de contrôle dans l'aléatoire). J'allais de temps en temps au STA, le service technique de l'artillerie, à St Thomas d'Aquin. Il y avait un vieux colonel tout petit, tout gris, comme une souris, qui conservait les archives. La joie que je pouvais avoir à lire le Mémorial de l'artillerie, et les articles probabilistes, les débats, y compris d'ailleurs des articles de Dumas, mais de beaucoup d'autres : qu'est-ce que c'est vraiment que la probabilité ? De temps en temps, ces types, quand ils arrivaient à avoir 4 ou 5 galons, ils avaient le temps de réfléchir et se disaient «mais bon sang de bonsoir, qu'est-ce que ça veut dire ? » Et des débats très... en particulier est déjà présent le problème de ce qui va devenir les querelles bayesiennes ou anti-bayesiennes. Les types se demandent «ce qu'on nous a appris comme probabilité des causes - puisque c'est comme ça qu'on parlait à l'époque - qu'est-ce que cela signifie ? Qu'est-ce que c'est que les probabilités a priori, dans quels cas a-t-on le droit d'en mettre, dans quels cas n'en a-t-on pas le droit? Et la probabilité uniforme, est-elle bien synonyme de l'ignorance totale ?» Tout ça, ce sont des problèmes qui se posaient...

D. BAYART : Maurice Dumas continue à se poser ces questions.

G.-TH. GUILBAUD : Oui, mais ce qui est remarquable c'est qu'il n'était pas tout à fait seul. De son tempérament, il était batailleur. Il l'est peut-être encore resté... je ne l'ai pas $\mathrm{vu}$ depuis longtemps. Les batailleurs donnent souvent l'impression d'être isolés... ce n'est pas vrai. Quand on regarde sur la longue durée, disons cinquante années du Mémorial, on s'aperçoit qu'il y a des gens intelligents qui se posent de vrais problèmes.

En particulier la présence des problèmes, et je l'ai vécu aussi, dans mon apprentissage. J'ai appris «probabilité des causes »- et puis j'ai appris qu'il y avait des gens qui n'étaient pas à leur aise là-dedans, et j'ai appris le théorème de Bayes, et que c'était important ; et même, en un sens, la discipline que j'ai reçue, c'est qu'on ne peut pas s'en dispenser. Que si on s'en dispense, c'est qu'il y a un truc quelque part et qu'il 
faut aller y voir. De sorte que la grosse affaire d'Abraham Wald, la redécouverte que dans un certain domaine, les seules stratégies admissibles sont les stratégies bayesiennes, nous avait donné chaud au coeur.

\section{LA TAYLORISATION TRANSFORME LE RAISONNEMENT STATISTIQUE EN « RÉGLEMENTS}

DE MANEUVRE »

G.-TH. GUILBAUD : Est-ce que ces problèmes préoccupaient les théoriciens, ou bien les praticiens qui avaient des loisirs ? Parce qu'il ne faut pas oublier qu'en statistique, l'urgence de la fabrication industrielle fait que l'on n'a pas toujours le temps de se poser des questions, et que, dans beaucoup de domaines industriels - nous revoilà au taylorisme - et aussi militaires - on aime bien les règlements de manoeuvres. C'est pourquoi la diffusion de la pratique des tests dits « de signification » a été si rapide : ça se présente comme un règlement. Le maréchal des logis, il aime bien avoir le règlement: «on fait comme ci, on fait comme ça», il ne faut pas chercher à comprendre.

Que cela puisse être appliqué par des gens peu qualifiés ou formés, c'est taylorien aussi bien que militaire. Et c'est évidemment un barrage au progrès intellectuel, parce qu'une fois qu'un règlement est adopté... une fois qu'un contrat sur épreuves de recette a été signé, on ne veut plus rien savoir, c'est comme ça que ça se passera.

D. BAYART : Et la production utilisable de la théorie, c'est des tables; celles de Dodge et Romig pour le contrôle de réception ont été rééditées pendant la dernière guerre...

G.-TH. GUILBAUD : Oui, bien sûr.

\section{LES MULTIPLES ASPECTS DU « CLIENT » AUQUEL S'ADRESSE LE STATISTICIEN}

G.-TH. GUILBAUD : Alors, vous demandez : quelle a été l'attitude des clients par rapport au hasard ? Ce que j'ai retiré de mon combat concernant la recherche opérationnelle : attention au niveau. On a réussi à voir des hommes importants, de grands messieurs, mais il faut savoir leur parler, c'est difficile. Un slogan disait « un prix Nobel à côté du Président $\gg . .$.

Sur cette question de "gestion de l'aléatoire », disons : il ne suffit pas que, dans une entreprise, les techniciens aient des trucs, et tout ça. Il faut aussi que, à la tête, pour les grandes décisions, il y ait des gens qui aient compris que cette science existait. Ça, c'est plus difficile. Je me souviens de réunions organisées sous l'influence de Darmois, groupant des PDG de très haut niveau, pour qu'on leur explique de quoi il s'agissait dans le domaine de la gestion du risque. Alors, un homme comme Massé était fort respecté, Darmois était déjà... et des gens comme moi, ça ne pèse pas lourd. Donc, comment leur parler, voilà ce que me suggère votre formulation. Mais le mot «client » est ambigu.

D. BAYART: À vrai dire, nous pensions plutôt au «risque du client», le risque d'accepter un lot de qualité insuffisante, symétrique du « risque du fournisseur », qui est celui de refuser un lot de qualité acceptable. Comment cette notion est-elle née ? Dumas dit que c'est lui qui l'a inventée...

G.-TH. GUILBAUD : Oui, il dit souvent cela ; il ne faut pas toujours le croire, mais c'est quelquefois vrai... «Le client » me fait songer à ceci : dans les grandes entreprises (ah ! il y a eu aussi les pétroliers avec lesquels j'ai eu des relations, la SHELL spécialement, sur des questions de programmation pour le raffinage), il y a des innovations. Les 
innovations technologiques proprement dites, le raffinage par exemple, ne posent pas trop de problèmes, elles sont admises. Mais là il y avait des innovations dont on se demandait si elles étaient d'ordre plutôt méthodologique que technologique. Il y avait un souci de l'équipe dirigeante pour comprendre de quoi il s'agissait, de quoi on parlait. Chez les pétroliers, c'était assez réceptif. Par «client»-j'entends mes clients - c'était l'aspect hiérarchique de la question : ce n'était pas la même chose de parler au Directeur général entouré de son état-major, à un directeur de service, à un ingénieur de fabrication, à un ingénieur commercial. Et le langage doit être adapté. Il faut essayer de se rendre compte - et ce n'est pas facile - du type de responsabilités, du temps qu'ils peuvent consacrer à cela. Tout cela, c'est un problème qu'on ne nous enseigne pas à l'Université. Et ça fait partie du boulot de la statistique ; pourquoi ? parce que justement elle est en devenir, en perpétuel mouvement.

\section{Y A-T-IL DES SPÉCIFICITÉS DE LA RÉFLEXION PROBABILISTE ET STATISTIQUE EN FRANCE ? APERÇUS SUR QUELQUES PERSONNALITÉS}

D. BAYART : La question suivante est : «L'utilisation des statistiques dans l'industrie est-elle en retard en France par rapport à la Grande-Bretagne, aux États-Unis, à l'Allemagne ? Si tel est le cas, ce processus a-t-il des racines anciennes ou est-il relativement récent (années 1920...) ? ».

G.-TH. GUILBAUD : «Le retard en France », je ne sais pas ; en tout cas, s'il y en a un, c'est un problème relativement récent. Il faut voir cela sur la longue durée : l'histoire de la statistique, c'est au bas mot 250 ans, alors il y a des hauts et des bas. C'est très difficile d'avoir des vues qui ne soient pas journalistiques, historiographiques et superficielles, d'avoir des vues réelles sur : comment les choses évoluent quand elles évoluent lentement.

Vous vous interrogez sur «la différence entre la France, l'Angleterre et l'Allemagne... », mais il ne faudrait pas oublier l'Italie. Dès que je suis entré en statistique, un certain nombre de gens plus avancés que moi me disaient: «Ah! Attention, il y a les Italiens ». Les Italiens : d'abord, ils écrivent en italien entre les deux guerres. Mais il se trouvait que j'aimais beaucoup la langue italienne, que je la pratiquais, je suis allé en Italie quelques fois. Il y avait dans l'enseignement des grands noms : Cantelli, Castelnuovo, Gini, de Finetti...

Ah ! Je pense à un théoricien assez étonnant, Jean-André Ville. C'est lui qui a réussi en 37-38 à démolir complètement la fausse théorie des probabilités inventée par von Mises, la fameuse théorie des collectifs. Il a fait sa thèse sur le sujet. Et après il a été ingénieur à l'Alsacienne. Bouzitat l'a bien connu, il a travaillé avec lui, parce qu'il s'est beaucoup préoccupé de théorie des jeux, de l'application du calcul des probabilités. Il a écrit des choses très importantes concernant la probabilité dans la revue Câbles et transmissions, dans le genre de ce qui, à cette époque-là, s'appelait la cybernétique. La probabilité dans les transmissions : ça aussi, c'est un domaine intéressant, la théorie de l'information, comme on a dit à une certaine époque, l'entropie, la néguentropie. Dans ce domaine, un certain nombre d'ingénieurs ont eu des contacts avec le calcul des probabilités.

Il y a un homme qui connaît bien cela, mais ça vous écarterait de votre sujet, c'est Elie Roubine, sur la théorie du signal. Il a été professeur au CNAM et il a joué un grand rôle dans l'enseignement, aux ingénieurs et autres, aux Télécom par exemple : le signal aléatoire, les processus de Wiener-Lévy, vous voyez le genre. C'est un très bon théoricien, mais il a eu des contacts avec les milieux d'ingénieurs. C'est pourquoi je 
pense à lui. Mais c'est un domaine un peu différent, quoique Abraham Wald le connaissait bien aussi.

D. BAYART : Savez-vous quelque chose d'une mission en Angleterre à la Libération, sur les méthodes statistiques de contrôle de qualité, dirigée par Szolem Mandelbrojt ${ }^{8}$ ?

G.-TH. GUILBAUD : Non, et ce que faisait Mandelbrojt dans l'affaire, je ne sais pas... À la Libération, il y avait une certaine pagaille, alors un certain nombre de gens... Il fallait ne pas être suspect de quoi que ce soit. Si on avait une autorité, on vous demandait de faire des tas de choses ; on les faisait, quoi. C'étaient des gens honnêtes, c'était pour le bien de la patrie, alors on y allait, mais pas forcément... Une espèce de patronage, parce que je ne crois pas que Mandelbrojt, l'ancien, se soit jamais beaucoup intéressé aux... Est-ce qu'il y a eu une influence de Paul Lévy, qui est un géant de l'histoire de la probabilité, et qui a enseigné à l'École polytechnique pendant des années ? Est-ce qu'il y a eu parmi ses auditeurs, de temps en temps, quelqu'un qui comprenait ce qu'il disait ? Il aurait dû y en avoir... il ne parlait pas avec une grande éloquence, c'est sûr, mais, tout de même, c'était très respectable, sa pensée, sa façon de voir. Et c'était un très bon probabiliste. Alors, le fait qu'il était à l'X aurait dû lui donner quelques chances, dans un auditoire aussi sélectionné, d'avoir quelqu'un qui l'écoute. Est-ce que c'est arrivé ? Et comment se fait-il que les probabilités ne se soient pas davantage diffusées dans la société française, avec un gars de cette trempe, et à ce poste important?

D. BAYART: Il n'est jamais cité comme a pu l'être Darmois, par les hommes de statistique.

G.-TH. GUILBAUD : Ce n'était pas un homme d'action, c'est sûr. Darmois était un homme d'action. Lévy, c'était la probabilité qui l'intéressait, mais il avait une façon de présenter les travaux mathématiques probabilistes qui était extrêmement habile, très sûre, très forte, très profonde en même temps. Il a découvert des tas de choses prodigieusement intéressantes.

J'avais beaucoup d'estime, de vénération même, pour René Roy. Parce que vraiment il avait des idées profondes et voyait loin. Il n'a pas beaucoup écrit. Il a été le berger de l'économétrie française pendant longtemps. Darmois a été le premier directeur du labo d'économétrie du CNRS parce qu'il fallait un universitaire, Roy était ingénieur des Ponts. C'était l'ancien régime... avant 68. J'ai été le premier, je crois, à me faire élire dans une faculté de droit sans être docteur en droit: on a trouvé des biais juridiques pour me faire nommer professeur associé. Il fut une époque où les règles administratives, pour pouvoir dépenser quatre sous, c'était très difficile: il fallait vraiment avoir les sacrements idoines, et pas d'une autre couleur !

\section{LES SPECIFICITÉS SECTORIELLES DU LANGAGE STATISTIQUE}

D. BAYART : Pendant la guerre et après, Fréchet a fait beaucoup d'efforts pour faire connaître les probabilités et statistiques, pour faire communiquer les différents groupes concernés (artilleurs, actuaires, agronomes, biologistes, démographes, économistes, ingénieurs, etc.).

\footnotetext{
${ }^{8}$ Professeur au Collège de France, mathématicien «pur » très peu concerné par les applications, d'où le commentaire de Guilbaud. (cf. «Entretien à bâtons rompus avec Szolem Mandelbrojt (1899-1983)», par Benoît Mandelbrot (qui est son neveu), publications du Séminaire d'Histoire des Mathématiques de l'Université de Paris, 6,1985, 1-46).
} 
G.-TH. GUILBAUD : L'idée de Fréchet était un peu militaire, qu'il faut imposer un vocabulaire... C'est aussi un peu l'idée de l'AFNOR, qu'il faut imposer, imposer... Il faut quand même laisser les gens un peu souffler... Imposer des normes de fabrication ou de publication du chiffre, je suis tout à fait d'accord, mais le vocabulaire... quand le vocabulaire va titiller les profondeurs... Les commissions : il y a des gens qui aiment être commissaires, nous les appelons des policiers. Il en faut, bien entendu, mais il ne faut pas, tout de même, qu'ils la ramènent un peu trop fort... Parce que, il faut bien distinguer : si j'achète du café ou de la marmelade, il faut que l'étiquette soit en effet calibrée pour que je ne sois pas couillonné. C'est vrai. Par contre, dans le domaine des probabilités, où quelquefois ça va toucher des profondeurs quasiment métaphysiques, il faut éviter de patauger là-dedans avec de gros godillots d'adjudant. Enfin, ce n'est pas possible. Il ne me vient pas d'exemple dans la tête, mais j'en ai vu quelquefois, de ces gens qui voulaient... On peut essayer de suggérer du vocabulaire; on a quelquefois du succès, quelquefois on n'en a pas... Il m'est arrivé au moins trois ou quatre fois l'expérience de militer pour un nouveau vocabulaire. Des fois, ça réussit, et puis des fois ça ne réussit pas.

D. BAYART : Est-ce que les différences de vocabulaire technique entre les artilleurs, les actuaires, etc... étaient gênantes pour la communication?

G.-TH. GUILBAUD : Mais c'est comme quand on va en Italie : ils parlent italien. On s'y fait, ce n'est pas terrible. Prenez par exemple « la fourchette» des artilleurs, qui est un exemple tout à fait banal : cela demandait 30 secondes - et puis il fallait revenir le lendemain pour s'en souvenir, mais une fois qu'on sait, eh bien, on sait ce que ça veut dire. Le mot « écart probable », aussi utilisé par les artilleurs : évidemment, il paraît un peu biscornu, mais il ne faut pas leur imposer de l'appeler «écart médian ». Ils ont dit pendant des siècles « écart probable », eh bien ! qu'ils continuent.

Ainsi, la dégradation de l'idée de fourchette me paraît quelque chose, du point de vue philologique, extrêmement intéressant. La fourchette des artilleurs était quelque chose de bien précis, on savait ce que ça voulait dire : c'était 4 fois l'écart probable, qui est un écart médian, qui n'est pas l'écart type. Bien sûr, c'est emmerdant, mais c'est comme ça.

Et puis c'est devenu ce que vous savez, dans les sondages, c'est-à-dire n'importe quoi et rien du tout. La plupart des gens - et ça m'a beaucoup frappé, parce que c'est dans le cours de ma vie, qui pourtant est courte à l'échelle de l'histoire, que j'ai vu cette oscillation qui consiste à évacuer complètement la notion de probabilité. Ils pensent qu'avec un intervalle, disons « de confiance », on est sûr que la valeur est dedans. Alors, à quoi bon les probabilités, si on est sûr! C'est ça que les gens pensent. Et pour leur extirper cette idée de la tête, ce n'est pas à coups de vocabulaire seulement qu'on y arrivera.

Quoique pendant tout le temps que le mot de fourchette a été utilisé dans l'artillerie française, et ça doit dater, pas de Napoléon mais de peu de temps après ; ça doit dater de la Restauration, il faut voir les règlements de l'artillerie, c'est intéressant. J'ai rêvé naguère, quand je fréquentais les militaires, de faire une anthologie de tous les textes militaires, depuis 1830 à peu près, peut-être même un peu avant, qui mettent en jeu une probabilisation du tir. C'est très rigolo: on voit les précautions que les gens prennent pour défendre l'idée de probabilité contre son évacuation presque obligatoire. C'est un domaine dans lequel l'expérience de l'artilleur met en garde contre l'évacuation. C'est vrai que si l'artilleur se croit sûr de quoi que ce soit, il est foutu. Donc il faut qu'il garde constamment présente l'idée d'aléa, qu'il ne l'expulse pas, malgré les écarts-types, les fourchettes et les règlements de manoeuvre. C'est délicat, ça, et ça se fait plus ou 
moins bien. Et c'est intéressant du point de vue militaire, parce qu'il y a des niveaux : le manuel pour les officiers, et le manuel pour les gradés et subalternes : et on ne parle pas le même langage, bien entendu.

\section{LES ANNÉES 1950 : L'AFCET, LES COLLOQUES INTERNATIONAUX}

Une partie de ma biographie, c'est aussi l'AFCET, qui ne s'appelait pas comme ça. Mon sujet, c'était la gestion économique générale, pas le contrôle de fabrication. Mais comme le contrôle de fabrication était déjà installé dans certains milieux, on le donnait comme exemple pour les autres tentatives dites de recherche opérationnelle. Les gens du contrôle de qualité donnaient, un peu comme les actuaires, l'impression de former... on n'aurait pas dit une mafia, mais... ils avaient leurs idées. Ils avaient leurs associations, et même une association internationale. Et quand il s'est agi de fusionner, ça a posé des problèmes.

La Société française de recherche opérationnelle (SOFRO, premier nom de l'AFCET) était une société parallèle ; à côté, il y avait les gens qui faisaient de l'automatisme, et puis ceux qui faisaient du calcul (qui est devenu de l'informatique). On voyait bien qu'il y avait des liens entre tout ça, à savoir qu'il s'agissait des nouvelles méthodes de l'organisation scientifique de la production industrielle, ou commerciale. Il y a eu des pourparlers, des tentatives de fusion, auxquels je n'ai d'ailleurs pas beaucoup participé.

Il y a eu plusieurs colloques importants dans les années cinquante. Deux colloques «Décision », un en 59, l'autre en 67, et un colloque «Économétrie » en 1952, qui a été moteur, mais c'était uniquement théorie du risque et de la décision. C'est là que Allais a fait sa communication contre Savage, ce qui a commencé à faire parler sur ce thème. Les textes de Massé et Morlat étaient importants, aussi. C'était la politique des colloques internationaux, on avait des sous pour faire venir des gens «bien » du monde entier : Marschak, Ragnar Frisch, de Finetti, Arrow... Gibrat, aussi, voilà un homme qui a joué un rôle pédagogique, mieux que Divisia. Lui aussi est un probabiliste, pas mal du tout, très homme du monde et très "homme important», mais en même temps un puits de science... Un ingénieur au corps des Mines, c'est quand même quelqu'un... Van Dantzig, c'était un homme étonnant, une espèce de prophète, il parlait des digues d'Amsterdam.

\section{LA VOIE MATHÉMATIQUE VERS L'À-PEU-PRÈS}

Je suis un amoureux de la science, et de toutes les sciences. La psychologie, pour moi, ça existe comme une attitude scientifique, pas de doute. La sociologie aussi. J'ai défendu toute ma vie l'idée qu'il n'y a pas que la mathématique. Oh diable ! s'il n'y avait que la mathématique, nous serions foutus depuis longtemps. La mathématique est très belle, elle est unique en son genre. Elle est incomparable, ce n'est même pas une science tellement c'est beau. Mais... des attitudes scientifiques dans des domaines variés : ils ont tous à profiter de la mathématique, je crois. J'ai défendu cela et je le défendrais encore maintenant, même sur mes tout à fait derniers jours. Je crois que la mathématique, à condition bien entendu de n'être ni maîtresse, ni servante, c'est plus subtil que ça, c'est un état d'esprit. C'est surtout avoir l'expérience de la mathématique. Ce n'est pas la connaissance des mathématiques, c'est avoir fait des mathématiques - et continuer d'en faire, comme on dit qu'on fait du sport, pour garder ses muscles en bon état, la circulation et le reste... De même, faire des mathématiques, ce n'est pas forcément publier des choses... En mathématique, il y a l'avantage qu'on peut inventer 
des trucs sans que personne le sache, si j'ose dire, sans avoir besoin de le publier, parce que c'est déjà connu. Mais je le réinvente sans savoir qu'il y a eu un Japonais, il y a 20 ans, qui a publié le même théorème quelque part. Il n'y a pas d'érudition en mathématique. Bon, pour les carrières, c'est important. Alors, Fréchet était marrant à cause de ça, peut-être qu'il avait souffert dans sa jeunesse, mais il était pointilleux sur les priorités : "Qui est-ce qui a publié le premier tel théorème, tel algorithme, c'est très important ». J'avoue qu'on a toujours rigolé un peu. Non, faire des mathématiques, c'est essentiel. Les gens dont j'ai parlé et dont vous voyez que je pense du bien, ce que je trouvais chez eux, c'est qu'on pouvait parler de mathématique car ils en avaient une certaine expérience - plus ou moins profonde, bien entendu, mais réelle. Et l'expérience de la mathématique, c'est une chose irremplaçable. Et il faut, semble-t-il, l'apprendre assez jeune, pas trop tard.

D. BAYART : En vous écoutant, je me demande dans quelle industrie on « faisait de la mathématique » en votre sens. Au CEA, dans le nucléaire, et à EDF ils étaient bien obligés d'avoir une approche probabiliste ?

G.-TH. GUILBAUD : À l'Alsthom, Ville avait une petite équipe qui faisait de la science. Pour le nucléaire, oui, ils y sont bien obligés, mais on pourrait dire « malheureusement », parce que tout le problème est que les approches probabilistes, ce n'est pas automatique. Il ne suffit pas de mettre du calcul des probabilités quelque part, il faut encore le prendre dans le bon sens. Et ce n'est pas facile, parce que les déviations sont permanentes. Alors comment peut-on, sans... Moi, c'est une chose qui m'intéresserait, si j'avais le loisir et la force de faire de l'histoire : comment se prémunir... ? Maintenant je vois bien quels sont les dangers, les chutes, qui sont essentiellement ceux-ci : on commence par faire du calcul des probabilités, et au bout d'un certain temps on l'évacue. Alors, il y a des gens qui se prémunissent, qui arrivent à se garantir contre cette facilité mentale, qui est très ancienne, qui est permanente...

D. BAYART : En fiabilité, on raisonne par taux de défaillance. On multiplie des taux de défaillance, que l'on considère quasiment comme des variables certaines.

G.-TH. GUILBAUD : Là, il y a un autre problème important. La probabilité, quelque part, est représentée par un nombre, ou des nombres, enfin peu importe. Est-ce que cette connaissance $\langle\mathrm{p}=1 / 2 »$ ou $\langle\mathrm{p}=\sqrt{\pi / 6} \gg$ est certaine ? Il y a des gens qui n'aiment pas qu'on pose ce problème-là, parce que pour eux c'est de la philosophie. Et il y en a d'autres qui sont très inquiets. Alors, peut-on rendre floue - pour parler le langage de certains - la connaissance des probabilités ? Et au moyen de quelles techniques ? Parce qu'il ne suffit pas de dire « je pense que la probabilité est $1 / 2$, je n'en suis pas très sûr, mais peu importe ». C'est une première attitude, acceptable. Deuxième attitude : «Ah ! Est-ce que je peux mettre autour de cette probabilité un intervalle... ? », et alors ça commence. Ca devient important et impressionnant: qu'est ce qu'une connaissance incertaine d'une probabilité ? Est-ce que ça tient la route ? Nous y revenons toujours, c'est toujours le thème - je suis vieux, je radote par conséquent, mais quand même, jusqu'à mon dernier souffle... : c'est l'attitude de l'esprit humain devant l'à-peu-près. Thème qui n'a pour le moment qu'une seule porte de sortie: la mathématique, la construction mathématique. Sinon, on est dans le brouillard. Alors, ça ne veut pas dire que c'est mauvais et qu'il faut mettre tous les gens en prison, mon Dieu, non ! Mais il faut faire très attention que parler de l'à-peu-près dans les prémisses d'un raisonnement, c'est extrêmement délicat car on ne contrôle pas l'incertitude finale, on ne peut pas.

D. BAYART : Dans les calculs des taux de défaillance des systèmes, par exemple dans le rapport Rasmussen sur les risques nucléaires, on utilise des chiffres dont la signification 
est très contestable, notamment des taux de défaillance des opérateurs humains. Mais les praticiens disent qu'il leur faut aboutir à des chiffres, nécessaires pour comparer les différentes options, et qu'il vaut mieux cela que pas de chiffre du tout.

G.-TH. GUILBAUD : Les exigences de la pratique... ce n'est pas faux, en un sens. C'est vrai que quelque part, dans un calcul de probabilités, il y a des probabilités initiales, on n'en sortira pas, ce n'est pas possible qu'il n'y en ait pas. Et elles ne sont pas données de toute éternité, elles sont choisies. Or cela met en branle des tas de réflexes épouvantables; il y a des gens qui tombent presque malades de cette aventure : la menace du subjectivisme en probabilité. Mais ce n'est pas comme cela que se pose le problème. Le problème, c'est qu'il y a des probabilités initiales, on n'en sortira jamais. Et des probabilités finales. Il faut qu'il y ait les deux. Or on essaye de camoufler les probabilités initiales et d'évacuer les probabilités finales, sous prétexte qu'elles sont toutes petites. À ce compte-là, il n'y a pas besoin de faire du calcul des probabilités.

Dans le domaine actuariel, je le reconnais, il y a eu des précautions verbales et mentales considérables pour éviter ces catastrophes, qui sont de faire du calcul des probabilités tout en ayant l'air de ne pas en faire, ce qui est de l'hypocrisie. Notez que dans le milieu des assurances, tout le monde ne se rend pas compte de la signification des calculs... Il y en a beaucoup pour qui « c'est comme ça, et on marche ».

Il y a aussi le domaine des phénomènes boursiers, dont j'ai parlé avec Allais, avec Mandelbrot le jeune. Y a-t-il eu vraiment des tentatives de modèle probabiliste soigné ? Il semble bien, et ça vaudrait la peine de regarder. Il ne faut pas oublier que l'un des plus grands probabilistes français, Bachelier, c'est par là qu'il a commencé. Malheureusement, il faisait des fautes de calcul. La carrière de Bachelier a été épouvantable, mais c'est un très bon probabiliste, et quelques-uns de ses écrits sont d'une très grande profondeur. C'était un universitaire, peut-être ancien élève de l'ENS. Il a réussi à être prof d'université, mais il y a eu une catastrophe à sa thèse. Elle est racontée partiellement par l'un de ses dévots, Benoît Mandelbrot, dans l'un de ses livres sur les fractales. Il met à la fin quelques notices biographiques sur les gens pour qui il a du respect dont Paul Lévy, George Kingsley Zipf et autres. Bachelier a eu, comme on dit, des malheurs, et n'a pas eu la carrière à laquelle il avait droit, pour des raisons obscures. Il était probabiliste et il a écrit des choses admirables - au milieu, d'ailleurs, d'un fatras... il écrit vers 1900. Je parle de lui parce qu'il a eu contact avec la spéculation boursière.

La Bourse, c'est une autre piste. C'est un jeu, il y a des aléas, pas de doute. Est-ce que des gens ont phosphoré de façon intelligente là-dessus ? Je ne sais pas. Il doit y avoir des conseillers scientifiques à la Bourse, dont quelques-uns ont une formation actuarielle, par exemple. Allais et Mandelbrot s'intéressaient à la chose. Mandelbrot avait, il me semble, reçu une commande de l'industrie, peut-être sur le cours du coton. 